Volume 8, No.5, September - October 2019

International Journal of Advanced Trends in Computer Science and Engineering

Available Online at http://www.warse.org/IJATCSE/static/pdf/file/ijatcse101852019.pdf

https://doi.org/10.30534/ijatcse/2019/101852019

\title{
Legal Advisory System based on K-Nearest Neighbor (K-NN) neural network for Iraqi Law
}

\author{
Amar A Sakran ${ }^{1}$, Dr. Nawaf Rasheed Alharbe ${ }^{2}$, Dr. Intisar Al-Mejibli ${ }^{3}$ \\ ${ }^{1}$ University of Information and Communication Technology, Baghdad, Iraq, Email: amarabdraba@gmail.com \\ ${ }^{2}$ Department of Computer Science and Information, Community College, Badr, Taibah University, KSA \\ Email: nrharbe@taibahu.edu.sa; anawafrasheed@gmail.com \\ ${ }^{3}$ University of Information Technology and Communications, Baghdad, Iraq, Email: \\ dr.intisar.almejibli@gmail.com; dr.intisar.almejibli@uoitc.edu.iq
}

\begin{abstract}
In general, the procedures in many courts around the world, in-cluding Iraqi courts, need effort and time to be applied, which may influence the people daily routine negatively. Recently, the governments in some countries seek to reform these procedures with cooperation of existing security sector. Additionally, devel-oping in the information technology, facilities using artificial intelligent mechanism in the field of legal. This paper presents a Legal Advisory Decision Support System (LADSS) that has the ability to analyze the legal problems depending on knowledge base and taking the proper decision to the user, which could be (Judge, lawyer, client). Where the proposed system aims to smooth applying the procedures in courts, eliminate biases, min-imize the required time $\&$ effort and achieve justice. It takes the legal case as an input, and then it provides the court with the hearing scenario for each given legal case. This paper proposed a decision support system for IRAQI Law, the system was tested by many real legal cases and the results were promising.
\end{abstract}

Key words: Artificial intelligent mechanism, Decision Support System, legal reasoning, Back propagation, IRAQI Law.

\section{INTRODUCTION}

Any case in Iraqi courts or police stations needs time and procedures, which may be long, until the judge or responsible person takes a decision. This is attributed to many reasons related to the conditions of each case and the experience of the judge. Sometimes, the bias may affect the taking decisions, which leads to lack of justice or the taken decision maybe not the proper decision [1].

Few researchers investigated the legal cases with Artificial Intelligence (AI) mechanisms. The achieved studies can be classified into three classes: tackles specific section of law, estimate a decision, or simulate the court scenario. Although, these studies have contribution in this field, they did not presented a solution for achieving successful criminal justice system. Because they faced many challenges such as language of written law.

Basically, the language is regarded as the main issue in such system, as all the laws are written as consecutive sentences that covered all the details related to law. Representing these sentences in computer to be used by AI techniques is not an easy task especially, when these sentences are overlapped and interference with each other as existing in real law.

Currently, the AI techniques have been used in different fields such as medical, business and other computer applications [2, $3,4]$ that encourage implementing them in legal field.

The aim of this research is answer the question of how to achieve a criminal justice system for Iraqi courts and police stations by using the techniques of AI without delay. The main idea of paper is designing and implementing a criminal justice system based on AI techniques. The proposed criminal justice system tackles the issue of the language representation in AI mechanisms by using many techniques, which are described in section nine of this paper. The proposed system is named as Legal Advisory Decision Support System (LADSS). Where the Arabic language is used in writing the Iraqi Law. The proposed system has been trained on a real cases with their final decision based on using its knowledge base. It has the ability of analyzing the legal problems depending on the available knowledge base and taking the proper decision to user (Judge, lawyer, client). The proposed system takes the legal case as an input, and then it provides the court with hearing scenario for each given legal case. Furthermore, the system has the ability of determining the court decision and afford the legal clarification to user (Judge, lawyer, client).

\section{RELATED WORK}

The first who used the term artificial Intalegint (AI) and law was McCarty1977 and here we will use in our legal advisory system more than one strategy in machine learning approach like case based reasoning (CBR), rule based reasoning (RBR), and a hyper systems . 
The authors of [5] proposed a Hypo system which is an case-based reasoning system. Hypo system has been developed to addresses the trade secrets law. Likely, this system is well known and good documented among all the proposed systems of case-based reasoning. Hypo can analyses the case conditions in the trade law area and use case base to retrieves relevant cases to construct them as legal arguments". The author of [6] suggested Malicious Prosecution Consultant (MPC) system named as kowalski's ystem. MPC is a CBR system that deals with cases in the field of malicious prosecution" [25].

HELIC-II (Hypothetical Explanation constructor by Legal Inference with Cases by 2 inference engines) is a system that developed by the authors of [7]. This sytem is hybrid legal that operats in the domine of penal code and utilising the applied legal rules (the law) with cases (precedents).

The $\mathrm{PhD}$ dissertation in [8] presented a system named JUDGE. This system is a case based reasoning system that aims to simulate the sentencing achived by real-life judges on criminals through comparing current cases to previous cases with thier penalities. This sytem based on interviewing the judges to explor their opinion and procedures in specific hypothetical cases to conclude the influence factors.

The author of [9] developed a system named IKBALS that deals in the domine of workers compensation law. IKBALS is applied by combining the rule based and case based system" so it's a hybird system.

The authors of [10] suggested case-based reasoner system called as OPINE. OPINE operates in generic legal cases. This system affores estimation of likely case outcome by using only one function. This features makes it different from the afornmentioned CBR systems.

The authors of [11] proposed an earlier system called as Legal Expert System for Termination of Employment Review (LESTER). LESTER is a case-based reasoning system operates in a sepicific domin which is unjust discharge from employment under collective bargaining agreements".

Author of [12] proposed a fuzzy CBR system that deals with legal inference. This system is implemented by combining the fuzzy charactristics and case rules for the cases of legal contracting.

Authors of [13] developed a system called HILDA that extractes the knowledge by using Artificial neural networks (ANNs). It used the extracted knowledge to lead the rule inferences and cases retrieval" [10].

Another neuro-fuzzy approach in legal reasoning is developed by the author of [14]. This approach attempts to determine structure in precedent decisions in addition to sepisfying legal precedents.

SHYSTER is a system that developed by author of [15]. It is a case-based legal expert system which has the ability to act as a hybrid by linking with rule-based models. It can operats in multiple legal domains, such as the laws of Australian copyright aspects, contract, personal property and administrative.

TAXMAN is a system developed by author of [16] and its a rule-based system. It can perform a basic form of legal reasoning by classifying cases based on a particular category of statutory rules in the domain of law concerning corporate reorganization.

\section{MACHINE LEARNING}

The machine learning techniques in the computer science, contract with learning algorithms and are accomplished to handle multifaceted difficulties over a set of experimentation and can grow its performance mechanically [17].

The customary technique of the machine learning is divided into supervised, unsupervised and reinforcement learning. The supervised learning is knowledge by a straight judgement between the input named (actual network) and output named (desired output). Although unsupervised learning does not comprise any actual network, it be governed by associations amid the actual input without the assistance of a teacher [18].

\section{ADVISORY SYSTEMS}

The advisory system is the system that provides advice to decision-makers through its architectural. The system feeds from previous experience, previ-ous cases, and knowledge engineering that is feed to the knowledge base through which the process of inference, which is controlled by the monitoring agent, for the best advice. Investigations in advisory systems have determined that decision makers require identifying and framing the issue in order, they can make proper decisions [19] [29].

\section{ADVISORY SYSTEMS DECISION SUPPORT}

"Advisory systems give support to deci-sions of both classes intelligent and unstructured. They are having the char-acteristics of novelty, complexity, and open end. Additionally, unstructured decisions are characterized with contex-tual uncertainty, which resulting in in-creases the complexity of the decision-making process when combined expo-nentially [20] [21]. Figure (1) shows the architecture of advisory systems.

\section{CLASSIFICATION}

Classification is a process of splitting the objects into groups where all objects in a group have the same features. There are many examples of classifications and can be found in everywhere, such as supermarkets grouping similar ob-jects together so there is shelf for chick-en, fruits and vegetables, detergent products. Classifications facilitates the life, like in supermarket example, if objects were distributed randomly on shelf, it would make shopping tiring and bothering experience. Thus, most finan-cial institutions based on classification for their credit ratings, medicine uses it extensively for diagnosing diseas-es"[22].

Many proposed techniques uses the clas-sification that are applied in data mining with Neural Network (NN). Artificial $\mathrm{NN}$ are former $\mathrm{NN}$ and for simplicity, they will be referred to 
as NN. Based on learning technique, NN have two types: supervised where the output values are known in advance such as back propaga-tion (BP) algorithm and unsupervised where the values of output are not known such as clustering techniques [23].

NN have an architecture govern by the number of nodes, weights set between the nodes, training the network and evaluating the results. In addition to, activation function, learning rate, mo-mentum and pruning. Probably, $\mathrm{K}$ -Nearest-Neighbor Classifiers is regarded as the most popular $\mathrm{NN}$ algorithm, which is demonstrated in this paper [24].

\section{K-NEAREST-NEIGHBOR (KNN) ALGORITHM}

The k-nearest-neighbor technique was first defined in the early 1950s. It worked thorough assumed big training sets, and did not gain popularity until the 1960s when amplified computing power developed available. It has since been commonly used in the area of pattern recognition.

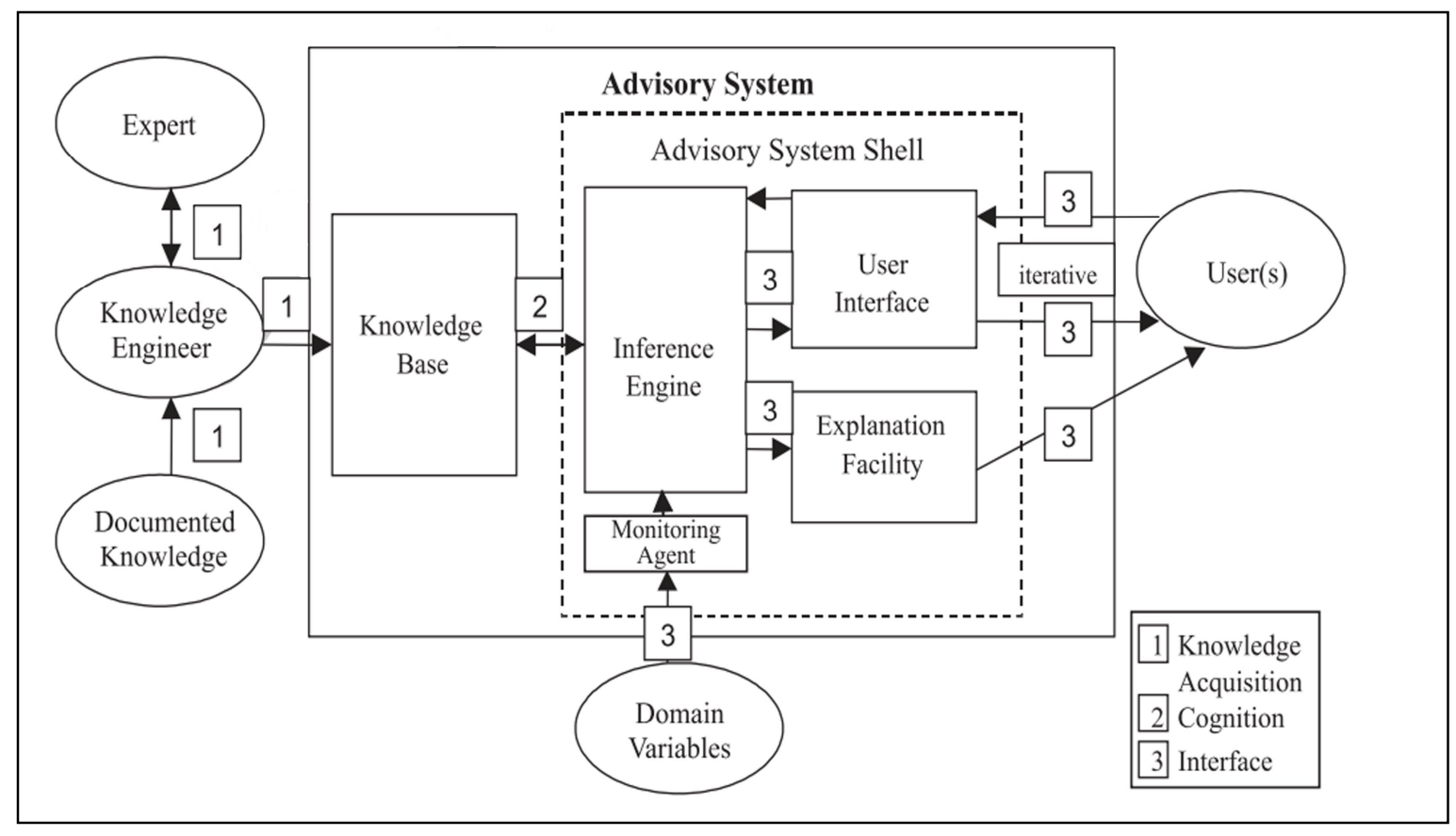

Figure 1: Advisory Systems Architecture. [24]

\section{NEURAL NETWORKS}

Over the last few years, the interest is increased in using NN's in many applications as they are being successfully applied in various domains, such as finance, medicine, engineering, geology and physics [25].

McCullock and Pitts created neuron in 1943 when they proved that neuron can have two states where these two states dependent on some threshold value [26]. The first artificial neuron model was presented by these two researchers according to Rojas [22]. Intelligent machines are established based on discovery of McCullock and Pitts. Basically, there are two main reason behind investigation the NN: first is attempt to understand the function of human brain and second is desire to create machines that have the ability of solving complex issues that traditional computers cannot solve them. When the structure of an issue is well analyzed and formed, traditional computers can perform better NN. Whereas in cases when issue has not well analyzed, NN can be used to learn from large set of data. NN capable of handles errors better than programs of traditional computers [30].
Nearest-neighbor classifiers are built on learning by similarity, that is, by associating an assumed test tuple with similar training.

The training tuples are defined by $\mathrm{n}$ attributes. Each tuple signifies a point in an n-dimensional space. In this way, all the training tuples are kept in an n-dimensional pattern space. When given a strange tuple, $\mathrm{k}$-nearest-neighbor classifier explorations the pattern space for the $\mathrm{k}$ training tuples that are closest to the unknown tuple. These $\mathrm{k}$ training tuples are the $\mathrm{k}$ "nearest neighbors" of the unknown tuple [27][31].

\section{FRAMEWORK OF PROPOSED SYSTEM}

Goal based agency is an appropriate process, has been used to achieve the goal of proposed system (LADSS).

Goal based agency is expanding abilities by utilizing goal information, which is used to describe the desired situations. Where these situations allow the agency in a way to choose from several possibilities that connects to the goal. Planning and search in this type of agencies are dedicated to do action 
sequences that achieve the agency goals. Figure (2) shows a flowchart of the proposed system (LADSS).

Part from the proposed system is related to deal with Arabic language as many such applications [28].
Step 1: remove all whitespace and dote.

Step2: message splitting into token.

Step3: store the token in one-dimensional array.

Step 4: save the result tokenization in array.

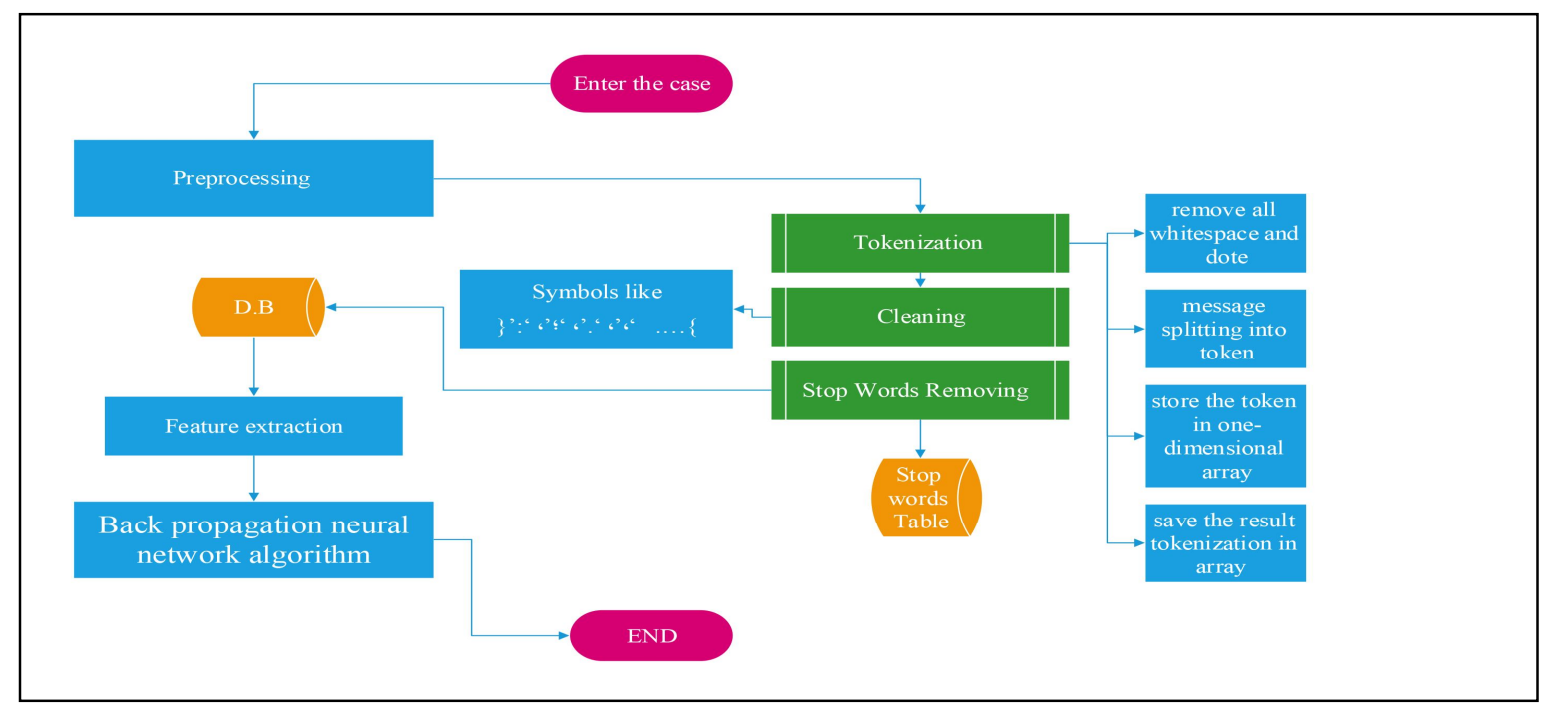

Figure 2: The Flow Chart of LADSS

The proposed Advisory decision support for criminal justice system has been implemented in four stages

There are four stages to achieve the goal

1- The Preprocessing

2- Feature extraction

3-k-nearest-neighbor ALGORITHM

4- The actuator, which represents the output.

\subsection{Preprocessing}

It is the second step in the proposed agency program. As the acquisition, input stage enters the data for preprocessing is regarded as the first step.

The entered data is various in their type and size because the $t$ types of article used in Iraqi Law are different. Some cases have details more than other cases; some cases have a lot of name complainant and complainer, dates, actions, places, etc. Preprocessing is the proper procedure to beat this issue.

Preprocessing remove the unwanted word like what that mentioned in the first paragraph and because we used a real case that write in Arabic language. This includes the following steps:

\section{a- Tokenization}

In order to transform a sentence into tokens (discrete words) the sentence passes through two phases: Sentence segmentation and Cleaning.

Tokenization or splitting the input is the procedure of breaking up a sequence of strings into pieces such as words, symbols, keywords, terms or attributes and other elements that Meaningful element are called tokens, in other words it is a form of text segmentation. Tokens act as an input for further processes like analysis and text mining.

The steps of tokenization as following:
The tokenization (or named Sentence Segmentation) separating the sentimental sentence to its words, which compose the sentence structure.

The proposed system analyzes sentence written in Arabic. As mentioned previously, the proposed systems use case for implementing the training phase

\section{b- Cleaning}

The previous phase produced a collection of words, symbols, punctuation marks, and numbers; therefore, it is necessary to remove unvalued words and symbols from the stored words obtained from the previous phase. Some of these words are expressions that contain HARKAT, which are do not have a value, because they are used for special purposes in Arabic.

\section{c- Stop Words Table}

Until now, the data stored in the table is large data set, and it contains many words that is not useful for the analyzing system which known as stop words.

A list of stop words stored in a tables in the database. Where each token in token table is compared with the content of the stop words database, if a token found in the stop words table then it will be removed.

\subsection{Feature Extraction}

The operation of feature extraction includes minimizing the amount of resources that are needed to explain a large set of data. Performing analysis for complex data faced a significant issue, which is the large of the number of involved variables. In general, analyzing large number of variables consumes a large space of memory and computation power; in addition, it may result in a poor performance of the classification 
algorithm in training samples and then generates poor new samples.

\section{a- Word-Based Features}

Knowing the length of the matrix and Calculation of the length of the array. It is one of the most important steps of the program, because its results are used in the statistical rocesses, on which many steps in the system depend.

Step1: This is done by passing each word of the array one by one, and reading the word whether it is within the rule table or not.

Comparing the read words with words in rule table will increase the count when reading each word to the end of the array.

The count results are sent to the database, in its own table with sending the name of the message (case) that is represented and sequentially.

Step2: Each word is taken and stored in a variable. Then pass on all matrixes, if there is a matching word that has been stored in the variable; it saves it in a counter after finish the array the last number is saved with the counter.

The process will be repeated on all words and from the first word to the last word. It sends the counter values to the data. With every word repeated. This gives us an advantage in determining the style of the case.

\subsection{K-Nearest Neighbor (K-NN)}

As is known for the algorithm KNN, one of the most famous algorithms of artificial neural networks, which are based on the basis of learning through similarity. Figure (3) shows

K-Nearest Neighbor (K-NN). They calculating the Euclidean distance between the cases in the testing phase and the cases in the training phase after the advantages of the input were extracted.

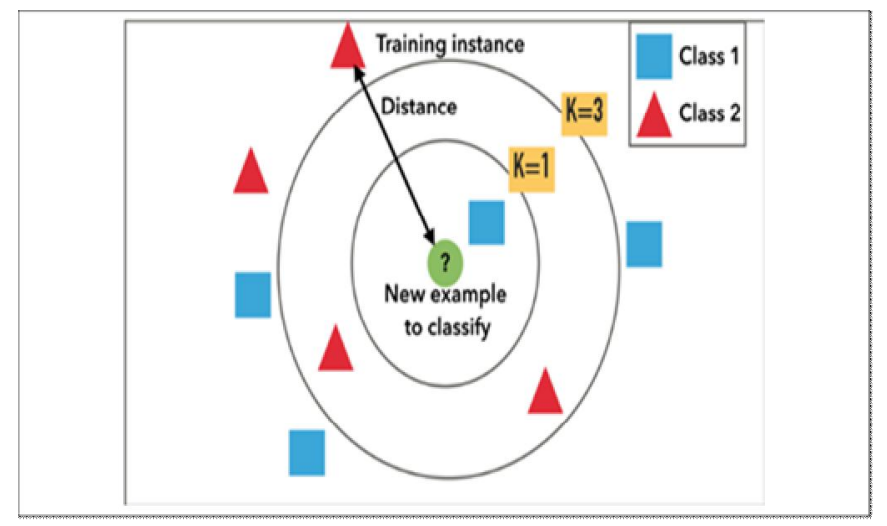

Figure 3: K-Nearest Neighbor (K-NN)

Euclidean distance with all cases stored in the knowledge base Generally the Euclidean distance formula is used to define the distance between two objects one in training phase and other in testing phase.

\subsection{The Actuator}

The actuator represents the output and it is the final stage in the proposed agency system. The actuator as stage will give an advice with suitable decision to the judge. This advice done according to the type of the case.

\section{RESULT}

The system is examined by using a set of real legal cases gained from KUT Court, where Kut is a city in the south of Iraq. 90 cases are used in this system where $80 \%$ used in training phase and $20 \%$ used in testing phase. The result from the proposed system was very good where it achieved (98\%) of accurate decision in testing phase. Accordingly, the system can get proper Advisory Judgment and a legal explanation to user for a case after training phase. It is impossible to compare the proposal results with results of the previous research, as it is different from them in technique and the applied cases types. Figure (4) shows the interface of the proposed system.

\section{CONCLUSION}

This paper developed Legal advisory decision support system, which is based on using AI mechanism. It includes four main steps: preprocessing, feature extraction, K-Nearest Neighbor (K-NN) and actuator.

The system achieved the rate of $98 \%$ as accurate decision. As a result, the system provides the judge, lawyer or client with correct advice and it reduces the required time for studying the case to taking the proper decision. In addition, it reduces the steps of traditional_work in courts.

Further, the system has the ability to conclude the legal case where all cases are stored in Knowledge Base (KB). Whereas the traditional work cannot overcome this issue.

This is the contribution of the new structure of the proposed legal knowledge base and the system.

Hence, the system is practical to use and gains high acceptance from users. 


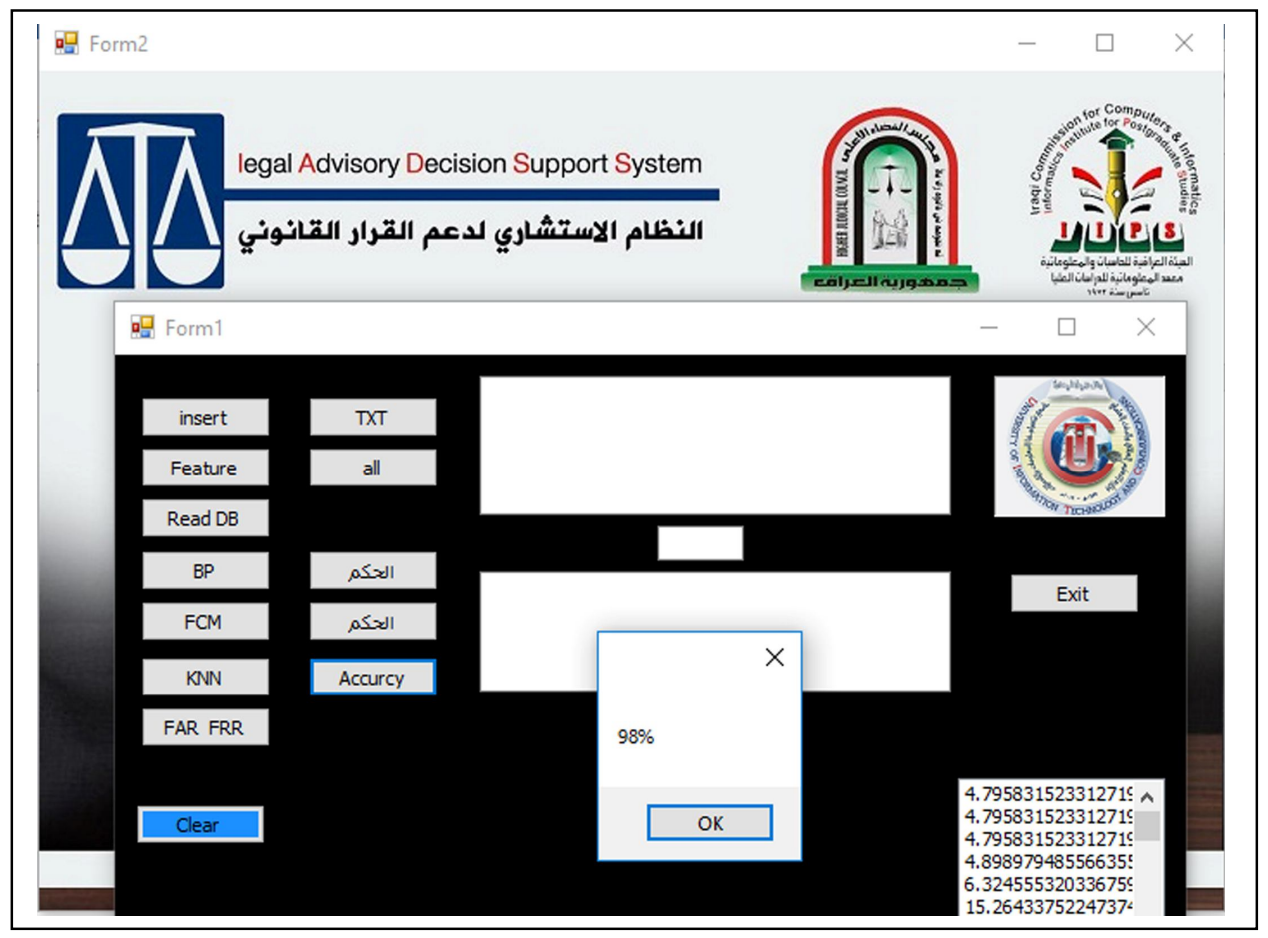

Figure 4: The Interface of the proposed System

\section{References}

1. N. Jasuja, P. Sehgal and Csi, "Procedural Law vs Substantive Law - Difference and Comparison Diffen", Diffen.com, 2018. [Online]. Available: https://www.diffen.com/difference/Procedural_Law _vs_Substantive_Law. [Accessed: 03-Aug- 2018].

2. D. H. Abd and I. S. Al-Mejibli, "Monitoring System for Sickle Cell Disease Patients by Using Supervised Machine Learning," 2017 Second Al-Sadiq International Conference on Multidisciplinary in IT and Communication Science and Applications (AIC-MITCSA), Baghdad, Iraq, 2017, pp. 119-124. doi: 10.1109/AIC-MITCSA.2017.8723006.

3. H. K. Hoomod, I. Al-Mejibli and A. IssaJabboory, "Optimizing SOM for cell towers distribution," 2017 Annual Conference on New Trends in Information \& Communications Technology Applications (NTICT), Baghdad, 2017, pp. 138-143. doi: 10.1109/NTICT.2017.7976136.

4. HODYCH, Oles; SHCHERBYNA, Yuriy; ZYLAN, Michael. SYNTHESIS OF SELF-ORGANIZING MAP AND FEEDFORWARD NEURAL NETWORK FOR BETTER FORECASTING. International Journal of Computing, [S.1.], p. 68-75, aug. 2014. ISSN 2312-5381.

5. K. Ashley, "Reasoning with cases and hypotheticals in HYPO", International Journal of Man-Machine Studies, vol. 34, no. 6, pp. 753-796, 1991.
6. A. Kowalski, "Case-based reasoning and the deep structure approach to knowledge representation", in Third International Conference on Artificial Intelligence and Law, England, 2018, pp. 21-30.

7. K. Nitta, Y. Ohtake, S. Maeda, M. Ono, H. Ohsaki and K. Sakane, "HELIC-II: Legal reasoning system on the parallel inference machine", New Generation Computing, vol. 11, no. 3-4, pp. 423-448, 1993.

8. W. Bain, "Case-Based Reasoning: A Computer Model of Subjective Assessment", PhD, Yale University New Haven, 1986.

9. J. Zeleznikow, G. Vossos and D. Hunter, "The IKBALS project: Multi-modal reasoning in legal knowledge based systems", Artificial Intelligence and Law, vol. 2, no. 3, pp. 169-203, 1994.

10. K. Lambert and M. Grunewald, "Legal theory and case-based reasons: The importance of context and the process of focusing", in Third International Conference on Artificial Intelligence and Law, England, 1991, pp. 191-195.

11. K. Lambert and M. Grunewald, "LESTER: Using paradigm cases in a quasi-precedential legal domain", in Second International Conference on Artificial Intelligence and Law, Canada, 1989, pp. 87-92.

12. K. Hirota, H. Yoshino, M. Q. Xu, Y. Zhu, X. Y. Li and D. Horie, "A fuzzy case based reasoning system for the legal inference," 1998 IEEE International Conference on Fuzzy Systems Proceedings. IEEE World Congress on Computational Intelligence 
(Cat. No.98CH36228), Anchorage, AK, USA, 1998, pp. 1350-1354 vol.2. doi: 10.1109/FUZZY.1998.686315.

13. P. A. Egri and P. F. Underwood, "HILDA: knowledge extraction from neural networks in legal rule based and case based reasoning," Proceedings of ICNN'95 - International Conference on Neural Networks, Perth, WA, Australia, 1995, pp. 1800-1805 vol.4. doi: 10.1109/ICNN.1995.488894.

14. J. Hollatz, "Neuro-fuzzy in legal reasoning," Proceedings of 1995 IEEE International Conference on Fuzzy Systems., Yokohama, Japan, 1995, pp. 655-662 vol.2. doi: 10.1109/FUZZY.1995.409754.

15. P. Koton, "Reasoning about evidence in causal explanation", in Proceedings of the Seventh National Conference on Artificial Intelligence, England, 1988, pp. 256-261.

16. J. Popple, "SHYSTER: A Pragmatic Legal Expert System", PhD., Australian National University, 1993.

17. M. I. Jordan and T. M. Mitchell, "Machine learning: Trends, perspectives, and prospects," Science: vol. 349 , no. 6245 , pp. $255-260,2015$. DOI: 10.1126/science.aaa8415.

18. A. Guido, Introduction to Machine Learning with Python. [S.1.]: O'Reilly Media, Inc., 2016.

19. D. Gregg and S. Walczak, "Auction Advisor: an agent-based online-auction decision support system", Decision Support Systems, vol. 41, no. 2, pp. 449-471, 2006.

20. L. Arendt, R. Priem and H. Ndofor, "A CEO-Adviser Model of Strategic Decision Making", Journal of Management, vol. 31, no. 5, pp. 680-699, 2005.

21. J. Aronson and E. Turban, "Decision Support Systems and Intelligent Systems", (7th Edition), Prentice-Hall, Inc. Upper Saddle River, NJ, USA 2004

22. X. Liu, D. Yang, and A. E. Gamal. "Deep neural network architectures for modulation classification", in 51st Asilomar Conference on Signals, Systems, and Computers, USA, 2017, pp. 4-3.

23. S. Yousaf, I. Shafi and J. Ahmad, "Features Selection and Optimized Neural Network Architecture for Modelling Flows in Solar Collectors," 2017 International Conference on Frontiers of Information Technology (FIT), Islamabad, 2017, pp. 247-252. doi: 10.1109/FIT.2017.00051.

24. B. A. Beemer, and D. G. Gregg., "Systems to Support Decision Making", Business School, University of Colorado, Denver, CO, USA, 2007, pp.361- 377, [Online] Available: https://www.semanticscholar.org/paper/Systems-toSupport-Decision-Making-Beemer-Gregg/1a9c47c2 81213f0be2679bbe0bdb6268a4c26bcd.
25. M. Negnevitsky, "Arti cial intelligence: a guide to intelligent systems", Addison-Wesley, ISBN 0321204662, 9780321204660, 2005.

26. A. Makarenko, "Toward development of neural networks with strong anticipation," 2017 IEEE First Ukraine Conference on Electrical and Computer Engineering (UKRCON), Kiev, 2017, pp. 1084-1087. doi: 10.1109/UKRCON.2017.8100417.

27. J. Han \& M. Kamber and Jian. Pei,'Data Mining Concepts and Techniques", Third Edition, Elsevier Inc. USA, 2012, pp 400-423.

28. V. L., Helen Josephine; S., Duraisamy. NOVEL PRE-PROCESSING FRAMEWORK TO IMPROVE CLASSIFICATION ACCURACY IN OPINION MINING. International Jounal of Computing, [S.1.], p. 234-242, dec. 2018. ISSN $2312-5381$.

29. Dalila B.M.M. Fontes, Paulo A. Pereira, Fernando A.C.C. Fontes," A Decision Support System for TV self-promotion Scheduling", International Journal of Advanced Trends in Computer Science and Engineering, Volume 8, No.2, March - April 2019. https://doi.org/10.30534/ijatcse/2019/06822019.

30. Jyoti Goyal, Bal Kishan, "Progress on Machine Learning Techniques for Software Fault Prediction", International Journal of Advanced Trends in Computer Science and Engineering, Volume 8, No.2, March - April 2019.

https://doi.org/10.30534/ijatcse/2019/33822019

31. Wai Yan Lai, Kuok King Kuok, Shirley Gato-Trinidad, Derrick, Kuo Xiong Ling, "A Study on Sequential K-Nearest Neighbor (SKNN) Imputation for Treating Missing Rainfall Data", International Journal of Advanced Trends in Computer Science and Engineering, Volume 8, No.3, May - June 2019. https://doi.org/10.30534/ijatcse/2019/05832019 